\title{
Drug-induced lupus with leukocytoclastic vasculitis: a rare expression associated with adalimumab*
}

\author{
Carolina Forte Amarante ${ }^{1}$ \\ Fátima Maria de Oliveira Rabay ${ }^{1}$ \\ Márcia Lanzoni de Alvarenga Lira ${ }^{1}$
}

\author{
Livia Mendes Sabia Acedo ${ }^{1}$ \\ Benedito do Espírito Santo Campos ${ }^{1}$ \\ Samuel Henrique Mandelbaum ${ }^{1}$
}

DOI: http:/ / dx.doi.org/10.1590/abd1806-4841.20153834

\begin{abstract}
A bstract: TNF alpha antagonist-induced lupus-like syndrome is a rare condition which predominantly affects women (4:1). The average age of onset is 46-51 years. It occurs after exposure to TNF alpha antagonist and disappears after discontinuation of such agents. The pathogenic mechanism for development of the TNF alpha antagonist-induced lupus-like syndrome is not fully defined. It is believed that the medication induces apoptosis, leading to an accumulation of nucleosomal antigens of apoptotic cells. This would cause autoantibodies to be produced by susceptible individuals. The most common cutaneous manifestations include maculopapular exanthem, malar rash, alopecia, photosensitivity and, more rarely, vasculitis.

Extracutaneous manifestations include: fever, weight loss, arthritis or arthralgia, myositis and hematological abnormalities. Antinuclear antibody may be positive in $80 \%$ of cases and anti-histone antibody is considered a disease marker for TNF alpha antagonist-induced lupus-like syndrome. Treatment corresponds to drug discontinuation. We report a rare case of sub-acute cutaneous lupus erythematosus with leukocytoclastic vasculitis induced by adalimumab in a 42-year-old patient.
\end{abstract}

Keywords: Exposure to biological agents; Tumor necrosis factor-alpha; Vasculitis, leukocytoclastic, cutaneous

\section{INTRODUCTION}

Tumor necrosis factor (TNF) alpha is a pro-inflammatory cytokine that is implicated in the pathogenesis of many chronic inflammatory diseases, including rheumatoid arthritis, ankylosing spondylitis, inflammatory bowel disease and psoriatic arthritis. ${ }^{1-9}$ TNF alpha antagonists were developed over a decade ago and have shown increasing success in controlling these diseases. ${ }^{6}$ One of the most common side-effects is the development of autoantibodies. Although the development of different autoantibodies is a common finding, only a few cases of drug-induced lupus erythematosus have been reported and they are rarely related to adalimumab. ${ }^{1-3,7,8}$ We report a rare case of Sub-acute cutaneous lupus erythematosus with leukocytoclastic vasculitis induced by adalimumab in a 42-year-old patient.

\section{CASE REPORT}

A 42-year-old male patient had a 10-year-history of psoriasis vulgaris and scalp psoriasis. After complaining of pain in the sacroiliac joint and knee, axial psoriatic arthritis was diagnosed. The laboratory tests showed elevated inflammatory markers, with negative antinuclear antibodies and rheumatoid factor. Methotrexate (15mg subcutaneously) plus narrowband UVB was introduced. However, given the decline in therapeutic efficacy, we decided to change to immunobiological therapy with adalimumab. The patient received adalimumab $40 \mathrm{mg}$ subcutaneously every two weeks plus topical calcipotriol, and showed good response to therapy. After 22 months of treatment, the patient complained of myalgia, arthralgia in the wrists and elbows, fever of 38 degrees and appearance of skin lesions. Dermatological examination revealed

Approved by the Advisory Board and accepted for publication on 04.08.2014

Study conducted at the Dermatology Service of the University Hospital of Taubaté - University of Taubaté (UNITAU) - Taubaté (SP), Brazil. Financial Support: None.

Conflict of Interest: None.

University of Taubaté (UNITAU) - Taubaté (SP), Brazil.

(C)2015 by Anais Brasileiros de Dermatologia 
discrete purpuric papules on the ends of the fingers and periungual telangiectasias. There were no other systemic signs. Laboratory evaluation showed elevated ESR, positive ANA at a titer of 1: 640 with homogeneous pattern and positive anti-histone antibodies (Figures 1, 2 and 3).

Histopathology of an erythematous-violaceous papule on the skin of the right hand dorsum showed a predominantly neutrophilic inflammatory infiltrate in the interstitium and in the wall of the superficial and deep capillaries. There was fibrinoid change in the wall of these capillaries and leukocytoclasia, consistent with leukocytoclastic vasculitis (Figures 4 and 5).

Based on the clinical and histopathological findings, we made the diagnosis of drug-induced subacute cutaneous lupus erythematosus. Adalimumab was discontinued and cyclosporine (300 mg daily) was prescribed, with no improvement. After three months with no response, we introduced etarnecept (50 mg weekly), with gradual reduction of cyclospo-

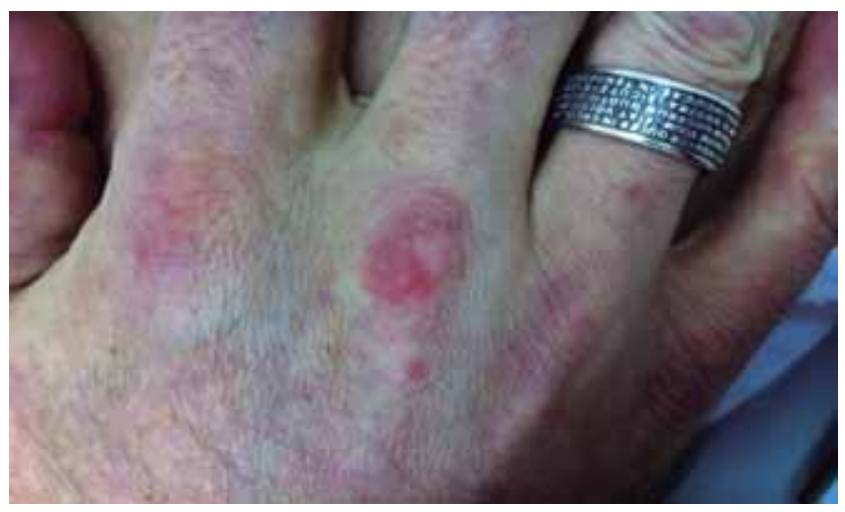

Figure 1: Psoriatic plaque on the third metacarpophalangeal joint, erythematous-violaceous papules on the right hand dorsum

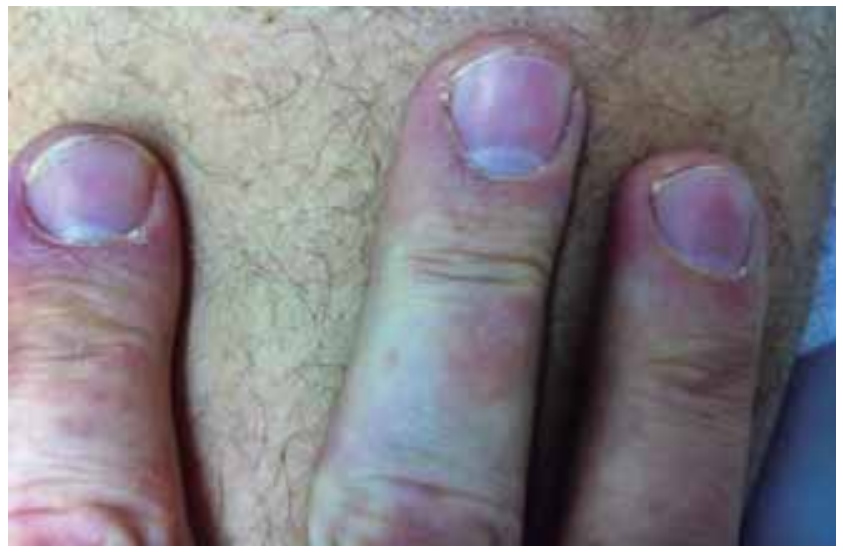

Figure 2: Purpuric papules on the distal ends of the right-hand fingers rine The patient had complete resolution of the symptoms, with no recurrence of psoriasis or lupus.

\section{DISAUSSION}

Drug-induced lupus-like syndrome (DILS) or, more specifically,TNF alpha antagonist-induced lupus-like syndrome is a rare condition which predominantly affects women (4:1). The average age of onset is $46-51$ years. It occurs after exposure to TNF alpha antagonist and disappears after discontinuation of such agents. ${ }^{1}$

The time to onset of lupus symptoms after initiation of TNF alpha inhibitors ranges from 10 days to 54 months. ${ }^{1}$ Infliximab and etanercept are the most common agents but adalimumab, more rarely, can also trigger the disease $\cdot{ }^{2-4}$ Adalimumab is the first fully human recombinant monoclonal IgG1 antibody which specifically binds to tumor necrosis factor alpha and neutralizes its biologic activity. ${ }^{4-7}$

The pathogenic mechanism for development of the TNF alpha antagonist-induced lupus-like syn-

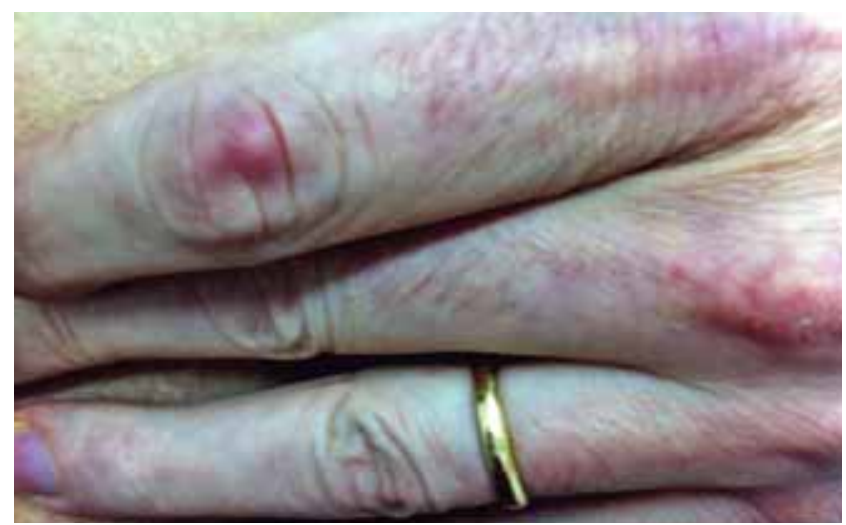

FIGURE 3: Erythematous-violaceous papule on the proximal interphalangeal joint of the left second finger

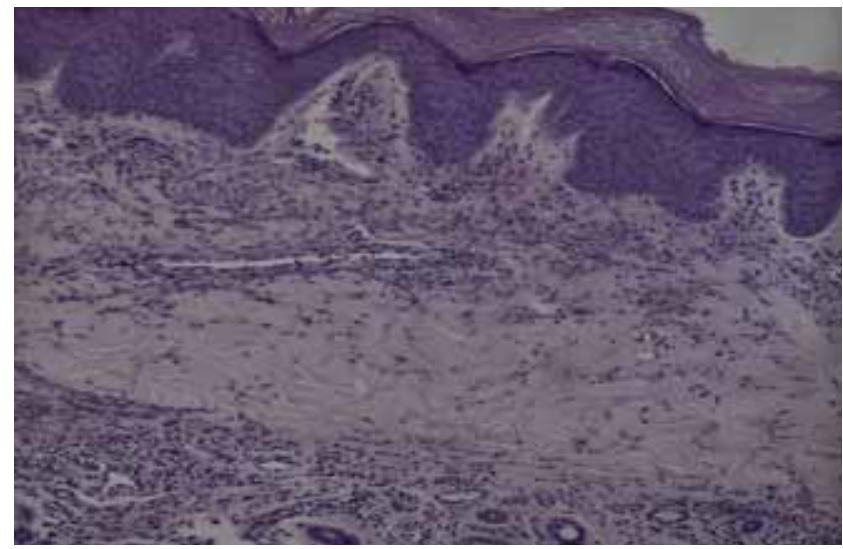

FIgURE 4: Predominantly neutrophilic inflammatory infiltrate in the interstitium and in the wall of the superficial and deep capillaries 


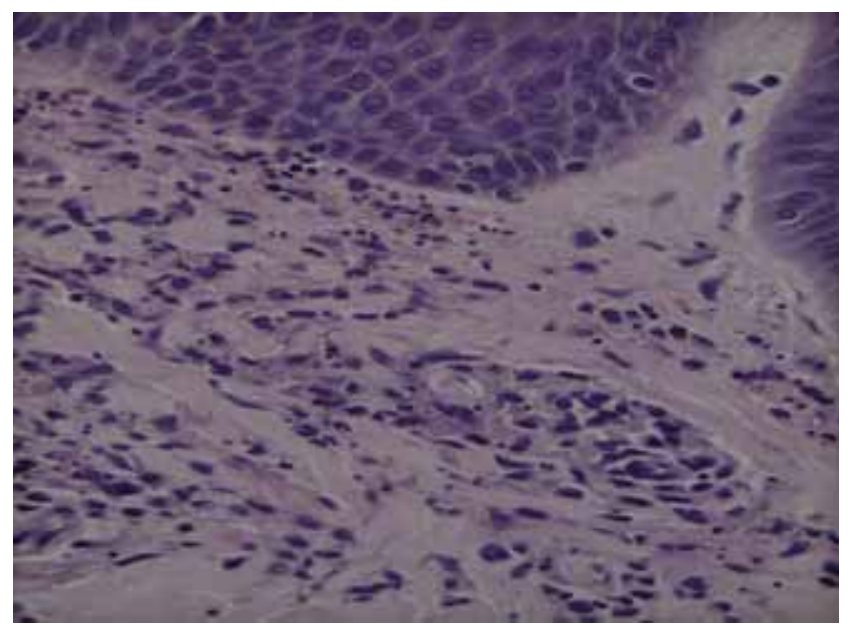

FiguRE 5: Fibrinoid alteration of the capillary walls, leukocytoclasia and red blood cell extravasation

drome is not fully defined. Some authors suggest that adalimumab, similarly to ultraviolet radiation in the pathogenesis of idiopathic lupus erythematosus, induces apoptosis, leading to an accumulation of nucleosomal antigens of apoptotic cells. This would cause autoantibodies to be produced by susceptible individuals. It was also suggested that the therapeutic inhibition of TNF-alpha would increase autoimmunity by inhibiting cytotoxic T lymphocytes. ${ }^{2}$

The drug-induced lupus-like syndrome can be subdivided into three clinical variants, namley: Drug-induced systemic lupus erythematosus (SLE), drug-induced sub-acute cutaneous lupus erythematosus and drug-induced chronic cutaneous lupus erythematosus. Clinical and laboratory characteristics of each of the aforementioned variants are shared by idiopathic lupus erythematosus. ${ }^{1}$

Systemic manifestations are: fever, weight loss, arthritis or arthralgia, myositis with elevated muscle enzymes, serositis and haematological disorders (ane- mia, leucopenia and thrombocytopenia). The most common cutaneous manifestations include maculopapular rash, malar rash, alopecia and photosensitivity. ${ }^{1,4,5}$ The most common presentation of vasculitis is the development of cutaneous lesions characterized by purpura, especially in distal limb, face and ears, and periungual telangiectasias. Histological analysis of the skin lesions usually shows cutaneous leukocytoclastic vasculitis, a pattern found in hypersensitivity vasculitis, involving superficial and deep dermal vessels, often with fibrin thrombi occluding vessels and associated frank necrosis. ${ }^{5}$

The least rigorous criteria for diagnosis requires one or more symptoms compatible with lupus erythematosus, ongoing exposure to a drug known to cause DILS, no prior history of lupus erythematosus, and resolution of symptoms when the offending drug is discontinued. ${ }^{1}$ There are no specific laboratory tests to diagnose DILS. Patients are screened for laboratory abnormalities found in idiopathic SLE. Serological tests include: autoantibodies, blood profile and renal function. ${ }^{1-3}$ ANA can be positive in $80 \%$ cases and the anti-histone antibody is considered a disease marker. However, it is less common in DILS (17-57\%). ${ }^{1}$

Treatment consists of drug discontinuation. Symptoms improve within 3 weeks to 6 months. Oral or injectable steroids, hydroxychloroquine or even immunosuppressive therapy such as azathioprine, cyclosporin, leflunomide, and methotrexate may be introduced in some cases. ${ }^{1-3.7}$

The complete resolution of symptoms after discontinuation of the drug, after a careful clinical and laboratory research, which excluded other etiologies, together with the histological pattern found, strengthen the diagnosis of drug-induced lupus erythematosus syndrome with leukocytoclastic vasculitis associated with the use of adalimumab. Given the potential severity of these reactions, early diagnosis and the choice of alternative treatment methods for a favorable disease outcome are of paramount importance.] 


\section{REFERENCES}

1. Williams VL, Cohen PR. TNF alpha antagonist-induced lúpus-like syndrome: report and review of the literature with implications for treatment with alternative TNF alpha antagonists. Int J Dermatol. 2011;50:619-25.

2. Vezzoli P, Violetti SA, Serini SM, Muratori S, Berti E, Crosti C. Cutaneous lupus erythematosus induced by adalimumab. J Dermatol. 2011;38:283-4.

3. Al-Niaimi F. Adalimumab-induced lupus erythematosus. Eur J Dermatol 2009;19:380.

4. Fiorino G, Danese S, Pariente B, Allez M. Paradoxical immune-mediated inflammation in inflammatory bowel disease patients receiving anti-TNF-a agents. Autoimmun Rev. 2014;13:15-9.

5. Perez-Alvarez R, Pérez-de-Lis M, Ramos-Casals M; BIOGEAS study group. Biologics-induced autoimmune diseases. Curr Opin Rheumatol. 2013;25:56-64.

6. Chew AL, Bennett A, Smith CH, Barker J, Kirkham B. Successful treatment of severe psoriasis and psoriatic arthritis with adalimumab. $\mathrm{Br} J$ Dermatol. 2004;151:492-6.

7. Exarchou SA, Voulgari PV, Markatseli TE, Zioga A, Drosos AA. Immune-mediated skin lesions in patients trated with anti-tumour necrosis factor alpha inhibitors. Scand J Rheumatol. 2009;38:328-31.

8. Martín JM, Ricart JM, Alcácer J, Rausell N, Arana G. Adalimumab-induced lupus erythematosus. Lupus. 2008;17:676-8.

9. Criado PR, Criado RFJ. Reações adversas a drogas: o espectro dermatológico na prática clínica. Barueri: Editora Manole LTDA; 2014. p. 430-6.
M AILING ADDRESS:

Carolina Forte A marante

A venida Granadeiro Guimarães, 270

Centro

12.020-130 - Taubaté - SP

Brazil

E-mail: carol_amarante88@hotmail.com

H ow to cite this article: Amarante CF, Acedo LMS, Rabay FMO, Campos BES, Alvarenga Lira ML, Mandelbaum $\mathrm{SH}$. Drug-induced lupus with leukocytoclastic vasculitis: a rare expression associated with adalimumab. An Bras Dermatol. 2015;90 (3 Suppl 1):S121-4 